\title{
Cultura Organizacional en la formalización de Microempresas en la ciudad de Huaral
}

\author{
Organizational culture in the formalization of Micro-enterprises in Huaral City.
}

Patricia Ramos ${ }^{1}$, Lucia Panta², Claudia Ramos ${ }^{3}$

\section{RESUMEN}

Objetivo: Determinar la relación que existe entre la cultura organizacional y la formalización de microempresas en el Mercado Modelo del distrito de Huaral. Material y Método: Se aplicó un diseño correlativo explicativo, con una muestra probabilística de 95 microempresarios. Se utilizó como instrumento un cuestionario de encuesta tipo escala Likert. Para la prueba de hipótesis se utilizó el coeficiente de correlación de Spearman entre las variables. Resultados: El 52,0\% de los encuestados afirmaron que existe un alto clima organizacional en los puestos que laboran y el $67 \%$ de los encuestados señalaron que el proceso de registro y lo relacionado a la formalización es alto. Se determinó la existencia de una relación positiva de alta a media entre los comportamientos observados, las normas, los valores dominantes y el clima organizacional con la formalización de microempresas; los cuales se evidenciaron en el coeficiente de Spearman de las hipótesis específicas, que resulto respetivamente $r=0,801, r=0,808, r=0,667, r$ $=0,622, r=0,748$. Conclusión: El estudio concluye que entre las variables cultura organizacional y formalización de microempresas en el mercado modelo de Huaral año 2019, existe una relación lineal estadísticamente significativa, considerable y directamente proporcional con un $\mathrm{pv}=0.000$, y un grado de correlación alto, con un coeficiente de Spearman de 0.813 .

Palabras claves: cultura organizacional, valores, formalización, proceso

\section{ABSTRACT}

Objective: To determine the relationship that exists between the organizational culture and the formalization of micro-enterprises in the Model Market of the Huaral district. Material and Method: An explanatory correlative design was applied, with a probabilistic sample of 95 microentrepreneurs. A Likert scale survey questionnaire was used as an instrument. For the hypothesis test, the Spearman correlation coefficient between the variables was used. Results: The $52.0 \%$ of the respondents affirmed that there is a high organizational climate in the jobs they work and $67 \%$ of the respondents indicated that the registration process and that related to formalization is high. Conclusion: The study concludes that among the variables organizational culture and formalization of micro-companies in the Huaral model market year 2019, there is a statistically significant, considerable and directly proportional linear relationship with $\mathrm{apv}=0.000$, and a high degree of correlation, with a Spearman coefficient of 0.813 .

Keywords: organizational culture, values, formalization, process

\section{INTRODUCCIÓN}

Las empresas son unidades que se adaptan a las condiciones sociales y económicas de un determinado ambiente, esto genera que las empresas desarrollen una cultura organizacional, la cual determina la orientación en el presente y futuro de la organización.

Según Fuentes (2012); la Cultura Organizacional es la unificación o mezcla de las creencias que está basada en significados creados por los individuos, producto de su interacción social que han sido naturalizados a través de un espacio temporal, los cuales se convierten en los elementos simbólicos y orientación de comportamiento. Por tanto, las empresas desarrollan su cultura organizacional sobre los valores, políticas, comportamientos, comunicación que se establecen en el interior de la organización.

En el Perú el proceso de transición de la informalidad a la formalidad en las microempresas, es uno de los procesos más difíciles de aceptar e incorporar a la cultura de los emprendedores. La resistencia a la formalización es reflejo de naturalizar la informalidad como parte de nuestra cultura. Los emprendedores han convivido más de 50 años, con la abrumadora cultura de la informalidad. (Gestión, 2014).

Se considera microempresa en el Perú, a toda unidad económica constituida por una persona natural o jurídica, que puede adoptar una forma de organización (E.I.R.L., S.R.L., S.A.), ésta puede dedicarse a la extracción, transformación, producción, comercialización de bienes o prestación de servicios. Este tipo de organización se caracteriza por no superar 150 UIT en ventas anuales y no superar los 10 trabajadores en promedio al año. Sin embargo, existe en una gran parte de organizaciones con características de microempresas que se encuentran la informalidad.

En el Perú existen 3,500 mercados de abastos, Lima congrega 1250 mercados, de los cuales el nivel de tributación. es insignificante en comparación al manejo económico que desempeñan estos mercados. Una clara señal de carencia de formalización, se calcula que cada stand de estos mercados vende aproximadamente 20,000 soles mensuales, y cumple con las características de microempresa, lo que revela que tienen capacidad tributaria, sin embargo, existe una resistencia a la formalización. Dentro de 1250 mercados de abasto de Lima, encontramos al Mercado Modelo de Huaral, un centro de abasto sumamente concurrido, tiene un promedio de 60 años al servicio del distrito de Huaral,

En estos últimos años una pequeña parte de las microempresas del mercado han migrado a la formalización; sin embargo, aún persiste cerca del setenta por ciento de stands del mercado son informales, es decir no tributan, no están registrados como 
organizaciones, ni tiene personal registrado en los organismos competentes. A pesar, de las campañas promovidas por los órganos competentes, se observa una apatía por parte de los emprendedores hacia la formalización.

Ante esta problemática, la investigación buscó esclarecer cómo se relaciona la cultura organizacional con la decisión de formalizarse en estos empresarios. y la dinámica entre la cultura organizacional y la formalización de las microempresas del mercado Modelo de Huaral, año 2019.

\section{MATERIALES Y MÉTODOS}

El diseño de la investigación fue de tipo correlacional y transversal. La técnica utilizada fue la observación.

La población estuvo constituida por los 1614 adscritos como socios al Mercado Modelo de Huaral y la muestra por 95 miembros adscritos al mercado Modelo de Huaral.
Las técnicas utilizadas para la recolección de datos fueron: la entrevista, la encuesta y la observación; el instrumento utilizado fue el cuestionario con valores en escala de Likert. El primer cuestionario contenía 20 ítems relacionado con la cultura organizacional, agrupadas en cuatro dimensiones: Comportamiento observados, normas, valores dominantes, filosofía. El segundo cuestionario contenía 20 ítems relacionado con la formalización empresarial, agrupadas en cuatro dimensiones: Sensibilización, Registro de Empresa, Incentivar Formalización, Cumplimiento. La información fue procesada en el software estadístico SPSS versión 22,0 .

\section{RESULTADOS}

Variable: Cultura Organizacional

\section{Tabla 1}

Niveles de comportamientos observados.

\begin{tabular}{cccccc}
\hline & Frecuencia & Porcentaje & $\begin{array}{c}\text { Porcentaje } \\
\text { válido }\end{array}$ & $\begin{array}{c}\text { Porcentaje } \\
\text { acumulado }\end{array}$ \\
\hline Válido & Bajo & 9 & 10,0 & 10,0 & 10,0 \\
& Medio & 29 & 30,0 & 30,0 & 40,0 \\
& Alto & 57 & 60,0 & 60,0 & 100,0 \\
& & & 100,0 & 100,0 & \\
\hline
\end{tabular}

En la tabla 1 se puede apreciar que el $60 \%$ de los encuestados del mercado Modelo de Huaral indicaron que hay un nivel alto en los comportamientos observados, el $30 \%$ un nivel medio y el $10 \%$ un nivel bajo.
En tal sentido, los datos estadísticos demostraron que el nivel de comportamiento observado en los encuestados del mercado Modelo de Huaral fue alto.

\section{Tabla 2}

Niveles de normas.

\begin{tabular}{lccccc}
\hline & Frecuencia & Porcentaje & $\begin{array}{c}\text { Porcentaje } \\
\text { válido }\end{array}$ & $\begin{array}{c}\text { Porcentaje } \\
\text { acumulado }\end{array}$ \\
\hline Válido & Bajo & 67 & 70,0 & 70,0 & 70,0 \\
& Medio & 14 & 15,0 & 15,0 & 85,0 \\
& Alto & 14 & 15,0 & 15,0 & 100,0 \\
& & & 100,0 & 100,0 & \\
\hline
\end{tabular}

En la tabla 2 se puede apreciar que el $70 \%$ de los encuestados del mercado Modelo de Huaral indicaron que hay un nivel bajo en el cumplimiento de normas, el $15 \%$ indicó que existe un nivel medio y el $15 \%$ un nivel alto. En tal sentido, los datos estadísticos demostraron que el nivel de cumplimiento de normas en los encuestados del mercado Modelo de Huaral fue bajo. 
Tabla 3

Niveles en valores dominantes

\begin{tabular}{lccccc}
\hline & Frecuencia & Porcentaje & $\begin{array}{c}\text { Porcentaje } \\
\text { válido }\end{array}$ & $\begin{array}{c}\text { Porcentaje } \\
\text { acumulado }\end{array}$ \\
\hline Válido & Bajo & 24 & 25,0 & 25,0 & 25,0 \\
& Medio & 5 & 5,0 & 5,0 & 30,0 \\
& Alto & 66 & 70,0 & 70,0 & 100,0 \\
& & & & \\
& Total & 95 & 100,0 & 100,0 & \\
\hline
\end{tabular}

En la tabla 3 se muestra que el $70 \%$ de los encuestados del mercado Modelo de Huaral indicaron que hay un nivel alto en los valores dominantes, el $25 \%$ indicó un nivel bajo y el $5 \%$ un nivel medio. En tal sentido, los datos estadísticos demostraron que el nivel de los valores dominantes en los encuestados del mercado Modelo de Huaral fue alto.

\begin{tabular}{lccccc}
$\begin{array}{l}\text { Tabla } 4 \\
\text { Niveles en filosofía }\end{array}$ & Frecuencia & Porcentaje & $\begin{array}{c}\text { Porcentaje } \\
\text { válido }\end{array}$ & $\begin{array}{r}\text { Porcentaje } \\
\text { acumulado }\end{array}$ \\
\hline Válido & Bajo & 24 & 25,0 & 25,0 & 25,0 \\
& Medio & 11 & 12,0 & 12,0 & 37,0 \\
& Alto & 60 & 63,0 & 63,0 & 100,0 \\
& Total & 95 & 100,0 & 100,0 & \\
\hline
\end{tabular}

En la tabla 4 se observa que el $63 \%$ de los encuestados del mercado Modelo de Huaral manifestaron que hay un nivel alto en la filosofía del negocio, el $25 \%$ indicó que hay un nivel bajo y el $12 \%$ indicaron que hay un nivel medio. En tal sentido, los datos estadísticos demostraron que el nivel en la filosofía del negocio en los encuestados del mercado Modelo de Huaral fue alto.

\section{Tabla 5}

Niveles de clima organizacional

\begin{tabular}{lccccc}
\hline & Frecuencia & Porcentaje & $\begin{array}{c}\text { Porcentaje } \\
\text { válido }\end{array}$ & $\begin{array}{c}\text { Porcentaje } \\
\text { acumulado }\end{array}$ \\
\hline Válido & Bajo & 19 & 20,0 & 20,0 & 20,0 \\
& Medio & 27 & 28,0 & 28,0 & 48,0 \\
& Alto & 49 & 52,0 & 52,0 & 100,0 \\
& & & & \\
\hline
\end{tabular}

En la tabla 5 observamos que el $52 \%$ de los encuestados del mercado Modelo de Huaral indicaron que hay un nivel alto en el clima organizacional, el $28 \%$ indicó que hay un nivel medio y el $20 \%$ un nivel bajo. En tal sentido, los datos estadísticos demostraron que el nivel de en el clima organizacional en los encuestados del mercado Modelo de Huaral fue alto. 
Variable: Formalización

Tabla 6

Niveles de sensibilización

\begin{tabular}{lccccc}
\hline & & Frecuencia & Porcentaje & $\begin{array}{c}\text { Porcentaje } \\
\text { válido }\end{array}$ & $\begin{array}{c}\text { Porcentaje } \\
\text { acumulado }\end{array}$ \\
\hline Válido & Bajo & 25 & 26,0 & 26,0 & 26,0 \\
& Medio & 50 & 53,0 & 53,0 & 79,0 \\
& Alto & 20 & 21,0 & 21,0 & 100,0 \\
& & & & \\
& Total & 95 & 100,0 & 100,0 & \\
\hline
\end{tabular}

En la tabla 6 se puede apreciar que el $53 \%$ de los encuestados del mercado Modelo de Huaral indicaron que hay un nivel medio en la sensibilización, el $26 \%$ que hay un nivel bajo y el $21 \%$ un nivel alto en la sensibilización. En tal sentido, los datos estadísticos demostraron que el nivel de sensibilización en los encuestados del mercado Modelo de Huaral fue medio.

\section{Tabla 7}

Niveles de registro de empresa

\begin{tabular}{lccccc}
\hline & Frecuencia & Porcentaje & $\begin{array}{c}\text { Porcentaje } \\
\text { válido }\end{array}$ & $\begin{array}{c}\text { Porcentaje } \\
\text { acumulado }\end{array}$ \\
\hline Válido & Bajo & 12 & 13,0 & 13,0 & 13,0 \\
& Medio & 19 & 20,0 & 20,0 & 33,0 \\
& Alto & 64 & 67,0 & 67,0 & 100,0 \\
& & & & \\
\hline
\end{tabular}

En la tabla 7 se muestra que el $67 \%$ de los encuestados del mercado Modelo de Huaral manifestaron que hay un nivel alto en registro de empresa, el $20 \%$ indicaron que hay un nivel medio y el $13 \%$ que hay un nivel bajo. En tal sentido, los datos estadísticos demostraron que el nivel en registro de empresa en los encuestados del mercado Modelo de Huaral fue alto.

Tabla 8

Niveles en incentivos

\begin{tabular}{lccccc}
\hline & & Frecuencia & Porcentaje & $\begin{array}{c}\text { Porcentaje } \\
\text { válido }\end{array}$ & $\begin{array}{c}\text { Porcentaje } \\
\text { acumulado }\end{array}$ \\
\hline Válido & Bajo & 22 & 23,6 & 23,6 & 23,6 \\
& Medio & 65 & 68,4 & 68,4 & 92,0 \\
& Alto & 8 & 8,0 & 8,0 & 100,0 \\
& & & & 100,0 & \\
\hline
\end{tabular}

En la tabla 8 se observa que el $68,4 \%$ de los encuestados del mercado Modelo de Huaral indicaron que hay un nivel medio en los incentivos, el $23,6 \%$ indicó un nivel bajo y el $8 \%$ que hay un nivel alto en los incentivos. En tal sentido, los datos estadísticos demostraron que el nivel de incentivos en los encuestados del mercado Modelo de Huaral fue medio. 
Tabla 9

Niveles de productividad.

\begin{tabular}{lccccc}
\hline & Frecuencia & Porcentaje & $\begin{array}{c}\text { Porcentaje } \\
\text { válido }\end{array}$ & $\begin{array}{c}\text { Porcentaje } \\
\text { acumulado }\end{array}$ \\
\hline Válido & Bajo & 10 & 10,9 & 10,9 & 10,9 \\
& Medio & 19 & 20,0 & 20,0 & 30,9 \\
& Alto & 66 & 69,1 & 69,1 & 100,0 \\
& Total & 95 & 100.0 & 100,0 & \\
\hline
\end{tabular}

En la tabla 9 se puede apreciar que el $69,1 \%$ de los encuestados del mercado Modelo de Huaral manifestaron que hay un nivel alto en la productividad, el
$20 \%$ un nivel medio y el $10,9 \%$ un nivel bajo. Estos valores demostraron que el nivel en la productividad en los encuestados del mercado Modelo de Huaral fue alto.

\section{Tabla 10}

Niveles en cumplimiento

\begin{tabular}{lccccc}
\hline & Frecuencia & Porcentaje & $\begin{array}{c}\text { Porcentaje } \\
\text { válido }\end{array}$ & $\begin{array}{c}\text { Porcentaje } \\
\text { acumulado }\end{array}$ \\
\hline Válido & Bajo & 24 & 25,0 & 25,0 & 25,0 \\
& Medio & 61 & 64,5 & 64,5 & 89,5 \\
& Alto & 10 & 10,5 & 10,5 & 100,0 \\
& & & 100,0 & 100,0 & \\
\hline
\end{tabular}

En la tabla 10 se observa que el $64,5 \%$ de los encuestados del mercado Modelo de Huaral indicaron que hay un nivel medio en el cumplimiento, el $25 \%$ un nivel bajo y el $10,5 \%$ un nivel alto en el cumplimiento. Por tanto en los encuestados del mercado Modelo de Huaral el nivel de cumplimiento fue medio.

\section{Prueba de hipótesis general}

Ho: La Cultura Organizacional no se relaciona con la formalización de microempresas en el Mercado Modelo de Huaral año 2019.

Ha: La Cultura Organizacional se relaciona con la formalización de microempresas en el Mercado Modelo de Huaral año 2019.

Estadística de prueba

Tabla 11

Correlación entre La Cultura Organizacional y la formalización de microempresas en el Mercado de Huaral año 2019

\begin{tabular}{|c|c|c|c|c|}
\hline & & & $\begin{array}{l}\text { Cultura } \\
\text { Organizacional }\end{array}$ & $\begin{array}{l}\text { Formalización } \\
\text { Empresarial }\end{array}$ \\
\hline \multirow{6}{*}{$\begin{array}{l}\text { Rho de } \\
\text { Sperman }\end{array}$} & & Coeficiente de correlación & 1,000 & $0,813^{* *}$ \\
\hline & $\begin{array}{l}\text { Cultura } \\
\text { Organizacional }\end{array}$ & Sig. (bilateral) & . & 0,000 \\
\hline & & $\mathrm{N}$ & 95 & 95 \\
\hline & & Coeficiente de correlación & $0,813^{* *}$ & 1,000 \\
\hline & $\begin{array}{l}\text { Formalización } \\
\text { microempresas }\end{array}$ & Sig. (bilateral) & 0,000 & . \\
\hline & & $\mathrm{N}$ & & 95 \\
\hline
\end{tabular}

** La correlación es significativa en el nivel 0,01 (2 colas). 
De acuerdo a los resultados obtenidos en la Tabla 11, se encontró que entre las variables Cultura organizacional y formalización de microempresas, existe una relación lineal estadísticamente significativa, considerable y directamente proporcional con un $p v=0,000$, que es menor a 0,05 y un grado de correlación alto, con un coeficiente de Spearman de 0,813.
Ho: Los comportamientos observados no se relacionan con la formalización de microempresas en el Mercado Modelo de Huaral año 2019

Ha: Los comportamientos observados se relacionan con la formalización de microempresas en el Mercado Modelo de Huaral año 2019.

Prueba de Hipótesis Específica 1

Tabla 12

Correlación entre la variable los comportamientos observados y la formalización de microempresas en el Mercado Modelo de Huaral año 2019

\begin{tabular}{|c|c|c|c|c|}
\hline & & & $\begin{array}{l}\text { Cultura } \\
\text { Organizacional }\end{array}$ & $\begin{array}{l}\text { Formalización } \\
\text { Empresarial }\end{array}$ \\
\hline \multirow{6}{*}{$\begin{array}{l}\text { Rho de } \\
\text { Sperman }\end{array}$} & & Coeficiente de correlación & 1,000 & $0,801^{\star *}$ \\
\hline & $\begin{array}{l}\text { Comportamientos } \\
\text { observados }\end{array}$ & Sig. (bilateral) & . & 0,000 \\
\hline & & $\mathrm{N}$ & 95 & 95 \\
\hline & \multirow{3}{*}{$\begin{array}{l}\text { Formalización } \\
\text { microempresas }\end{array}$} & Coeficiente de correlación & $0,801^{* *}$ & 1,000 \\
\hline & & Sig. (bilateral) & 0,000 & . \\
\hline & & $\mathrm{N}$ & 95 & 95 \\
\hline
\end{tabular}

** La correlación es significativa en el nivel 0,01 (2 colas).

En concordancia a los resultados obtenidos en la tabla 12 , se encontró que entre la dimensión comportamientos observados y formalización de las microempresas existe una relación lineal estadísticamente significativa, considerable y directamente proporcional con un $p v=0,000$, que es menor a 0,05 y un grado de correlación fuerte, con un coeficiente de Spearman de 0,801.

\section{Prueba de Hipótesis Específica 2}

Ho: Las normas no se relacionan con la formalización de microempresas en el Mercado Modelo de Huaral año 2019.

Ha: Las normas se relacionan con la formalización de microempresas en el Mercado Modelo de Huaral año 2019.

Estadística de prueba

\section{Tabla 13}

Correlación entre las normas y la formalización de microempresas en el Mercado Modelo de Huaral año 2019

\begin{tabular}{|c|c|c|c|c|}
\hline & & & Normas & $\begin{array}{l}\text { Formalización } \\
\text { Empresarial }\end{array}$ \\
\hline \multirow{6}{*}{$\begin{array}{l}\text { Rho de } \\
\text { Sperman }\end{array}$} & \multirow{3}{*}{ Normas } & Coeficiente de correlación & 1,000 & $0,808^{* *}$ \\
\hline & & Sig. (bilateral) & . & 0,000 \\
\hline & & $\mathrm{N}$ & 95 & 95 \\
\hline & \multirow{3}{*}{$\begin{array}{l}\text { Formalización } \\
\text { microempresas }\end{array}$} & Coeficiente de correlación & $0,808^{* *}$ & 1,000 \\
\hline & & Sig. (bilateral) & 0,000 & . \\
\hline & & $\mathrm{N}$ & 95 & 95 \\
\hline
\end{tabular}

** La correlación es significativa en el nivel 0,01 (2 colas).

De acuerdo a los resultados obtenidos en la Tabla 13 se encontró que entre las variables las normas y la formalización empresarial existe una relación lineal estadísticamente significativa, considerable y directamente proporcional con un $p v=0,000$, que es menor a 0,05 y un grado de correlación fuerte, con un coeficiente de Spearman de 0,808.

\section{Prueba de Hipótesis Específica 3}

Ho: Los valores dominantes no se relacionan con la formalización de microempresas en el Mercado Modelo de Huaral año 2019.

$\mathrm{Ha}$ : Los valores dominantes se relacionan con la formalización de microempresas en el Mercado Modelo de Huaral año 2019. 


\section{Tabla 14}

Correlación entre los valores dominantes y la formalización de microempresas en el Mercado Modelo de Huaral año 2019

\begin{tabular}{|c|c|c|c|c|}
\hline & & & $\begin{array}{l}\text { Valores } \\
\text { dominantes }\end{array}$ & $\begin{array}{l}\text { Formalización } \\
\text { Empresarial }\end{array}$ \\
\hline \multirow{6}{*}{$\begin{array}{l}\text { Rho de } \\
\text { Sperman }\end{array}$} & & Coeficiente de correlación & 1,000 & $0,667^{* *}$ \\
\hline & $\begin{array}{l}\text { Valores } \\
\text { dominantes }\end{array}$ & Sig. (bilateral) & . & 0,000 \\
\hline & & $\mathrm{N}$ & 95 & 95 \\
\hline & \multirow{3}{*}{$\begin{array}{l}\text { Formalización } \\
\text { microempresas }\end{array}$} & Coeficiente de correlación & $0,667^{* *}$ & 1,000 \\
\hline & & Sig. (bilateral) & 0,000 & \\
\hline & & $\mathrm{N}$ & 95 & 95 \\
\hline
\end{tabular}

${ }^{* *}$ La correlación es significativa en el nivel 0,01 (2 colas).

De acuerdo a los resultados obtenidos en la Tabla 14, se encontró que entre la dimensión valores dominantes y la formalización de microempresas, existe una relación lineal estadísticamente significativa, considerable y directamente proporcional con un $p v=0,000$, que es menor a 0,05 y un grado de correlación moderado, con un coeficiente de Spearman de 0,667.

Tabla 15

Correlación la filosofía y la formalización de microempresas en el Mercado Modelo de Huaral año 2019

\begin{tabular}{|c|c|c|c|c|}
\hline & & & $\begin{array}{l}\text { Valores } \\
\text { dominantes }\end{array}$ & $\begin{array}{l}\text { Formalización } \\
\text { Empresarial }\end{array}$ \\
\hline \multirow{6}{*}{$\begin{array}{l}\text { Rho de } \\
\text { Sperman }\end{array}$} & & Coeficiente de correlación & 1,000 & $0,622^{* *}$ \\
\hline & $\begin{array}{l}\text { Valores } \\
\text { dominantes }\end{array}$ & Sig. (bilateral) & . & 0,000 \\
\hline & & $\mathrm{N}$ & 95 & 95 \\
\hline & \multirow{3}{*}{$\begin{array}{l}\text { Formalización } \\
\text { microempresas }\end{array}$} & Coeficiente de correlación & $0,622^{* \star}$ & 1,000 \\
\hline & & Sig. (bilateral) & 0,000 & \\
\hline & & $\mathrm{N}$ & 95 & 95 \\
\hline
\end{tabular}

** La correlación es significativa en el nivel 0,01 (2 colas)

Según los resultados mostrados en la Tabla 15, se encontró que entre la dimensión la filosofía y la formalización de microempresas existe una relación lineal estadísticamente significativa, considerable y directamente proporcional con un $p v=0,000$, que es menor a 0,05 y un grado de correlación moderado, con un coeficiente de Spearman de 0,622.

\section{Prueba de Hipótesis Específica 5}

Ho: El clima organizacional no se relaciona con la formalización de microempresas en el mercado modelo de Huaral año 2019.

Ha: El clima organizacional se relaciona con la formalización de microempresas en el Mercado Modelo

\section{Prueba de Hipótesis Específica 4}

Ho: La filosofía no se relaciona con la formalización de microempresas en el Mercado Modelo de Huaral año 2019.

Ha: La filosofía se relaciona con la formalización de microempresas en el Mercado Modelo de Huaral año 2019. 
Tabla 16

Correlación el clima organizacional y la formalización de microempresas en el Mercado Modelo de Huaral año 2019

\begin{tabular}{|c|c|c|c|c|}
\hline & & & $\begin{array}{l}\text { El Clima } \\
\text { Organizacional }\end{array}$ & $\begin{array}{l}\text { Formalización } \\
\text { Empresarial }\end{array}$ \\
\hline \multirow{6}{*}{$\begin{array}{l}\text { Rho de } \\
\text { Sperman }\end{array}$} & & Coeficiente de correlación & 1,000 & $0,748^{* *}$ \\
\hline & $\begin{array}{l}\text { El clima } \\
\text { organizacional }\end{array}$ & Sig. (bilateral) & . & 0,000 \\
\hline & & $\mathrm{N}$ & 95 & 95 \\
\hline & \multirow{3}{*}{$\begin{array}{l}\text { Formalización } \\
\text { microempresas }\end{array}$} & Coeficiente de correlación & $0,748^{* *}$ & 1,000 \\
\hline & & Sig. (bilateral) & 0,000 & \\
\hline & & $\mathrm{N}$ & 95 & 95 \\
\hline
\end{tabular}

** La correlación es significativa en el nivel 0,01 (2 colas).

En concordancia a los valores indicados en la Tabla 16, se encontró que entre la dimensión clima organizacional y la formalización de microempresas existe una relación lineal estadísticamente significativa, considerable y directamente proporcional con un $p v=0,000$, que es menor a 0,05 y un grado de correlación alto, con un coeficiente de Spearman de 0,748.

\section{DISCUSIÓN}

En concordancia a los resultados obtenidos en la presente investigación, se evidenció que la dimensión comunicación tiene falencias en materia de aptitudes por parte de los administradores con respecto al procedimiento en ventas, donde se obtuvo un $30,6 \%$ de actitud positiva y un $69,5 \%$ de un aptitud negativa, es decir los administradores del puesto , no concordaron con los métodos aplicados por los trabajadores en las ventas, estos variaron en cada sector del mercado en coherencia al tipo de producto que se comercializa; así mismo la comunicación fue considerada con $60 \%$ como inapropiada por 'parte de los miembros de la micro empres (Tabla 12).

Al respecto, Calderón (2013); identificó las razones relacionadas a la cultura organizacional y su efecto en la estabilidad laboral, porque considera que una deficiente comunicación puede deteriorar la cultura organizacional. Por tanto, los datos obtenidos concuerdan con este autor confirmando que una deficiente comunicación se relación a la incorporación de la formalización.

En relación a la dimensión de normas, se evidenció dificultad con la comprensión y la débil difusión de las políticas de trabajo, ya que el $69,5 \%$ consideraba que no comprendían los aspectos normativos. Asimismo, un $56,8 \%$ manifestó que no existe normas de comportamiento, lo que indica que aún es incipiente la generación de normas en la organización. (Tabla 13). Los resultados corroboran lo afirmado por Calero (2018) que señala, que las microempresas no desarrollan objetivos claros debido a que existe una débil estructura normativa en sus organizaciones y por lo tanto sugiere que la implementación de cambios en la cultura organizacional está relacionada con el desarrollo de normas en la organización, puesto que considera que las normas claras va permitir alcanzar los objetivos de la organización.

En la dimensión valores dominantes, se estudió lo relacionado con la predominancia de la calidad, la investigación denotó que un $71,5 \%$ de los encuestados consideraron la importante calidad de producto. Asimismo, se evidenció $74,7 \%$ de ausentismo e impuntualidad (Tabla 14). La investigación demostró que el ausentismo y puntualidad son características que se pueden construir en las organizaciones, y de existir en forma negativa se puede mitigar su desempeño negativo, el cual tambien es reafirmado por Calderón (2013), que indica que la puntualidad debe ser fomentada dentro de la cultura organizacional.

En la dimensión filosofía, se evaluó lo relacionado con la apertura a nuevo personal, un $43,2 \%$ de los encuestados consideraron que los puestos donde ellos laboran existe una apertura por incorporar nuevo personal. El 68,4\% del personal con mayor experiencia mostraron predisposición por impartir sus experiencias, generando un clima colaborativo en las organizaciones (Tabla 15). Esto concuerda con lo indicado por Velásquez (2015) que establece una relación entre el cultura organizacional y compromiso organizacional y evidencia que el compromiso está asociado a la oportunidad que se le otorga al nuevo personal, esto va de la mano con la Etapa de inducción dirigida por el personal más antiguo que colabora en proceso de entrenamiento del nuevo personal.

En la dimensión clima organizacional, se estudió lo relacionado con el interés de los compañeros por el bienestar de su entorno, el 62,1\% de los encuestados afirmaron que perciben un interés por bienestar por parte de sus compañeros, de igual forma el $68,4 \%$ de los trabajadores percibieron la aceptación de sus aportes, y un $58,9 \%$ mostraron una actitud positiva frente a las situaciones nuevas, al respecto Calderón (2013); en su investigación donde describe lo factores de la cultura organizacional y la estabilidad laboral, considera que la materia de la cohesión laboral es generada por actitudes de compañerismo, es decir el interés que muestran entre compañeros por ayudarse en sus labores, lo que permite que tengan mejores habilidades para hacer frente a 
situaciones nuevas en la organización (Tabla 16). Los resultados permiten comprobar la relación lineal estadística significativa existente entre la dimensión cultura organizacional y formalización de microempresas del Mercado Modelo de Huaral año 2019.

\section{CONCLUSIÓN}

El estudio concluye que entre las variables cultura organizacional y formalización de microempresas en el mercado modelo de Huaral año 2019, existe una relación lineal estadísticamente significativa, considerable y directamente proporcional con un $p v=0.000$, que es menor a 0,05 y un grado de correlación alto, con un coeficiente de Spearman de 0.813 .

La resistencia a la formalización está asociado a una deficiente comunicación, los aspectos normativos no son claros ni estimulantes para el emprendedor, limitada identificación y difusión de los valores de la organización. Sin embargo, las dimensiones de filosofía y clima organizacional son promovidas por las microempresas como parte de su cultura organizacional. Por tanto, una cultura organizacional carente de comunicación eficiente, normatividad con bajo cumplimiento, valores incipientes, filosofía poco estimulante; son condiciones que retrae y generan la resistencia del micro empresario a ingresar a la formalidad.

\section{REFERENCIAS BIBLIOGRÁFICAS}

Calderón, A.Z.E (2013).La Cultura organizacional en la estabilidad Laboral. Tesis. Facultad de Humanidades. Universidad Rafael Landívar. Guatemala

Calero, G.F. (2018). La cultura Organizacional y el Desempeño del personal en las empresas procesadoras de atún de la provinciaa de Manabi (Ecuador). Tesis Facultad de Ciencias Administrativas. Universidad Mayor de San Marcos.Perú

Fuentes, D. A. E (2012). La cultura organizacional: Factor clave de éxito en las empresas del siglo XXI". Facultad de Ciencias Económicas. Universidad de Cartagena.

Gestión (2014). Controversia Económica: Informalidad: ha y que a t a c a r l a r a íz d e I problema.https://gestion.pe/blog/controversiaecon omica/2014/06/informalidad-hay-que-atacar-laraiz-del-problema.html/

Velásquez, F.E. (2015). Cultura Organizacional y Compromiso Organizacional en el Ministerio de la Producción Lima, 2015. Lima: Universidad César Vallejo 